\title{
Yeast Phosphofructokinase: Modeling Four Bacterial Tetramers to Act in Concert.
}

\author{
Carsten Peters ${ }^{*}$, Mirabela Rusu**, Stefan Birmanns** and Teresa Ruiz ${ }^{*}$ \\ *University of Vermont, Dept. of Molecular Physiology and Biophysics, Burlington VT 05405 \\ ** University of Texas at Houston, School of Health Information Sciences, Houston, TX 77030
}

Glycolysis is essential for the energy metabolism of living cells. Thus, phosphofructokinase (Pfk); a key regulatory enzyme of the glycolytic pathway, is expressed ubiquitously in all cellular organisms. The reaction catalyzed by Pfk, the phosphorylation of fructose 6-phosphate (F6P) to fructose1,6bisphosphate in the presence of ATP, represents the first irreversible step in the glycolytic pathway under physiological conditions. In prokaryotes, Pfk is a tetramer composed of four identical subunits of $35 \mathrm{kDa}$ in molecular mass and it is only regulated by two effectors (ADP as an activator and phosphoenolpyrovate as an inhibitor). On the other hand, eukaryotic-Pfks are substantially larger enzymes that exhibit a much more complex regulatory mechanism with over 20 effectors regulating their activity [1]. A deficiency or malfunction of Pfk can lead to muscle and non-muscle related diseases including hemolytic anemia, Alzeimer's disease and diabetes. Thus, a thorough understanding of the structure/function relationship of Pfk is needed to gain more insights into the mechanism of these diseases.

A combination of X-ray crystallography and biochemical data has yielded an understanding of the structure of the bacterial enzyme and of the conformational changes occurring between the active and inactive states (reviewed in [2]). However, of the eukaryotic enzymes only the structure of the Trypanosoma brucei Pfk, which shares $20-30 \%$ homology with bacterial-Pfk, is available [3]. The structure shows a striking similarity to its bacterial counterpart which serves as structural core modified by small insertions (loops) and larger N- and C-terminal extensions. The Pfk subunits of higher eukaryotic- have evolved from the bacterial gene by gene duplication, mutagenesis and fusion events, resulting in an $\mathrm{N}$-terminal catalytic region paired with a $\mathrm{C}$-terminal regulatory region [4]. Although to date no crystal structure has been published, several medium resolution Pfk 3D structures have been determined by both cryo-electron microscopy and negatively stained electron microscopy for the three yeasts (S. cerevisiae, S. pombe and P. pastoris) in various conformational states [5-9].

Our study focuses on both $S$. cerevisae and $S$. pombe Pfks which form octameric enzymes and are closer related to the mammalian Pfks than either the bacterial or T. brucei Pfk. The $\alpha$ and $\beta$ subunits of $S$. cerevisae and the $\alpha$ subunit of $S$. pombe (with mol. masses larger than $100 \mathrm{kDa}$ ) can be described as consisting of two bacterial subunits each fused together by a linker ( 50aa) with additional large N-terminal ( 200aa) and C-terminal ( 80aa) extensions. Bacterial-Pfk fits well into yeast-Pfk electron density maps derived by electron microscopy [7]. Surprisingly, the bacterial tetramer core (representing and $\alpha \beta$ dimer of $S$. cerevisae) remains intact, despite the big linker and the $S$. cerevisae Pfk octamer could be thought of as a complex comprising of 4 bacterial tetramers. However, the four bacterial cores account for less than 2/3 of the total molecular mass of the yeast Pfk octamer and cannot explain neither the interactions between the yeast tetramers nor the underlying structure for the sophisticated regulatory mechanism of eukaryotic Pfks. In the absence of a "good" crystal structure, we have used a combination of sequence alignment, homology modeling, and flexible fitting guided by our electron density maps together with available biochemical data to build models for the yeast Pfks. These models provide an interesting starting- 
point for understanding the concerted motions during the active to the inactive state transition.

\section{References:}

[1] Sols, A. (1981), Current Topics in Cellular Regulation 19: 77-101

[2] Evans P.R. (1992), Proceedings of The Robert A. Welch Foundation Conference on Chemical Research XXXVI. Regulation of Proteins by Ligands, Houston Texas 39-54.

[3] Martinez-Oyanedel J., McNae I.W., Nowicki M.W., Keillor J.W., Michels P.A.M., FothergillGilmore L.A. and Walkinshaw M.D. (2007), Journal of Molecular Biology 366:1185-1198.

[4] Poorman, R.A., Randolp A., Kemp, R.G. and Heinrikson R.L. (1984), Nature 309:467-469

[5] Ruiz T., Kopperschläger G. and Radermacher M. (2001) J. Struct. Biol. 136: 167-180.

[6] Ruiz T., Mechin I., Bär J., Rypniewski W., Kopperschläger G. and Radermacher M. (2003), J. Struct. Biol.143:124-134

[7] Barcena, M., Radermacher, M., Bär, J., Kopperschläger, G. and Ruiz, T. (2007) J. Struct. Biol. 159: 135-43.

[8] Benjamin, S., Radermacher, M., Bär, J., Edelmann, A. and Ruiz, T. (2007) J. Struct. Biol. 159:498-506.

[9] Benjamin, S., Radermacher, M., Kirchberger, J., Schöneberg, T., Edelmann, A. and Ruiz, T. (2009) J. Struct. Biol. 168:345-51.

[10] This work was supported by NIH grants R01 GM069551 and R01 GM069551-S1 (awarded to T.R) and benefited from developments supported by NIH grant R01 GM062968.

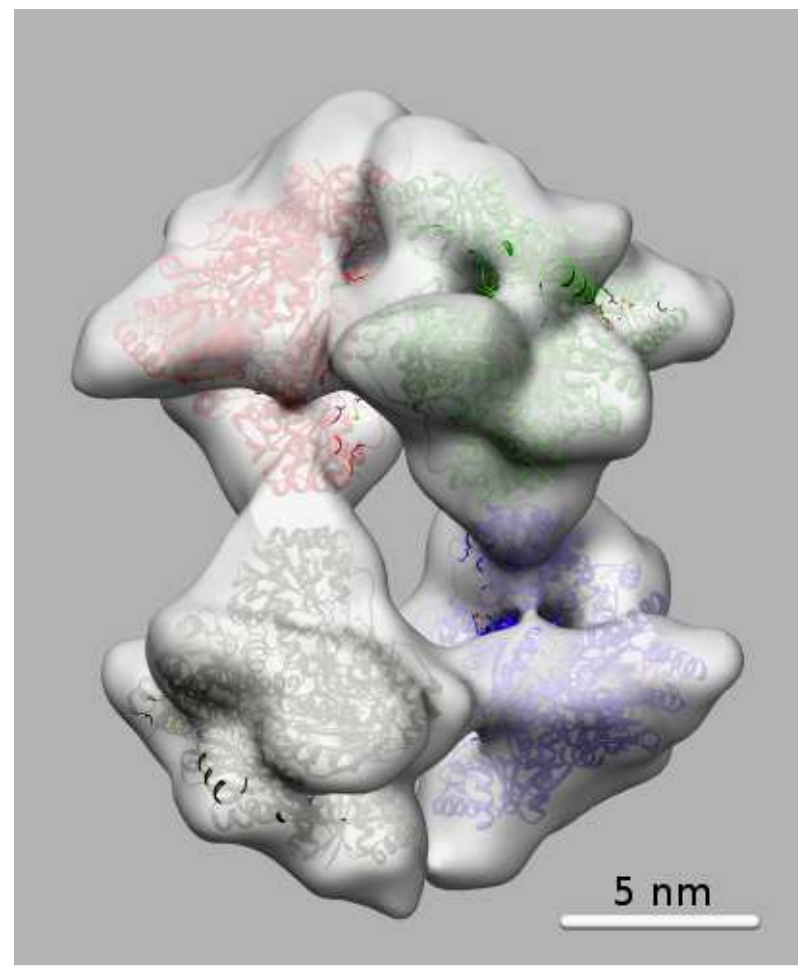

Figure 1: Cryo-electron microscopy map of the octameric S. cerevisae Pfk in the active state with four bacterial tetramers fitted in as ribbons (red, green, black and blue). 